\title{
1 Comparison of the Hydraulic Fracturing Water Cycle in China and North America:
}

\section{A Critical Review}

3 Cheng Zhong ${ }^{1,2^{*}}$, Ashkan Zolfaghari ${ }^{1}$, Deyi $\mathrm{Hou}^{2}$, Greg G. Goss ${ }^{3}$, Brian D. Lanoil ${ }^{3}$, Joel

$5 \quad{ }^{1}$ Department of Earth and Atmospheric Sciences, University of Alberta, Edmonton, Alberta,

$9 \quad{ }^{4}$ Department of Strategy, Entrepreneurship and Management, University of Alberta, Edmonton,

$11{ }^{5}$ Department of Civil and Environmental Engineering, The Hong Kong Polytechnic University,

$13{ }^{6}$ School of Energy and Environment and State Key Laboratory of Marine Pollution, City University of Hong Kong, Kowloon Tong, Kowloon, Hong Kong, China

15 *Corresponding author: Tel: 1-780-492-8019, email: alessi@ualberta.ca or czhong@ualberta.ca 


\section{Overview of flowback and produced water (FPW)}

18 Hydraulic fracturing (HF) operations in the U.S. typically require $280 \mathrm{~m}^{3}$ (10 ${ }^{\text {th }}$ percentile) to 23,000

$19 \mathrm{~m}^{3}\left(90^{\text {th }}\right.$ percentile) of fracturing fluid, consisting mostly of surface water or groundwater, to

20 fracture low permeability formations such as shales. ${ }^{1}$ After the HF process, $10 \%$ to $100 \%$ of the

21 total injected volume (TIV) of fracturing fluid returns to the surface as flowback and produced

22 water (FPW), ${ }^{2-4}$ which contains constituents of the fracturing fluid and their degradation products, ${ }^{5}$

23 hydrocarbons indigenous to the formation, reservoir rock constituents that have dissolved/reacted

24 with the fracturing fluid, and formation water which often has elevated salinity. ${ }^{1}$

\section{Additional description of unconventional development in China}

27 The Zhaotong (near the Sichuan Basin) and Yan-an (Ordos Basin) plays are other promising shale 28 gas development regions in the state plan, ${ }^{6}$ with other projected shale gas plays having only a few

29 test wells drilled at this stage. ${ }^{7}$ Unconventional oil production, including tight oil and shale oil 30 plays in areas such as the Ordos, Songliao, and Junggar Basins, accounts for less than $1 \%$ of annual 31 production in China, ${ }^{8,9}$ largely due to the clay-rich formations in these basins which make HF 32 operations more challenging. ${ }^{10}$

\section{Cases of HF-induced water stress in the U.S.}

35 Water use for shale gas production in Texas is concentrated in several counties, accounting for $36 \sim 80 \%$ of total water consumed in $2008 .{ }^{11}$ Furthermore, significant water consumption by HF 37 operations may cause water stress in certain streams during drier months in semi-arid regions such 38 as Arkansas. ${ }^{12}$ 
41 Recent trace metal profiles from China provide essential information for pollutant identification,

42 resource extraction, and the prevention of downhole facility clogging and corrosion (Table 2). For

43 example, isotopes of elements such as strontium and boron are good indicators of the original

44 depositional environments, which must be determined in order to identify FPW contamination. ${ }^{13}$

45 Elements such as iron, sulfate, barium, and strontium are related to infrastructure clogging and

46 corrosion; lithium (5-60 mg/L detected in FPW from Sichuan Basin ${ }^{13}$ ) is a valuable resource, ${ }^{14,15}$

47 and lead and cadmium are important elements in water toxicity assessments.

\section{The U.S. Geological Survey and FracFocus}

50 In North America, the U.S. Geological Survey (https://www.usgs.gov) has systematically

51 summarized FPW organic compounds identified in the literature or in publicly accessible

52 databases. Additionally, FracFocus (www.fracfocus.org and www.fracfocus.ca) provides basic

53 information about the injected chemical groups that can be referenced to support chemical

54 identification in FPW.

\section{Mechanics of reuse for the fracturing of new wells}

57 The reuse of FPW commonly involves separating large particles (mainly sand and drill cuttings)

58 using a desander, and then storing FPW in wastewater impoundments for a period of time to allow

59 for the sedimentation of suspended solids. ${ }^{16,17}$ Pre-treated FPW is subsequently mixed with water

60 and other chemical additives to produce new fracturing fluid. ${ }^{16}$

61

\section{FPW treatment for organics}


63 Organics in FPW may cause fouling of the treatment facilities and they are difficult to fully remove,

64 while recent evidence shows that organic compounds such as ethylene glycol and polyacrylamide

65 in FPW have synergistic effects in the removal of other FPW organic compounds such as di-(2-

66 ethylhexyl) phthalate during wastewater treatment. ${ }^{18,19}$

\section{Guidelines/standards for surface discharge of treated FPW in China}

69 In China, FPW discharge requirements follow the more general Integrated Wastewater Discharge

70 Standard (GB 8978-1996) or Environmental Quality Standards for Surface Water (GB 3838-

71 2002). ${ }^{20-23}$ Other relevant regulations in China include the Technical Specification for Oil

72 Production Wastewater Treatment (HJ2041-2014), and the Technical Specification for

73 Wastewater Treatment in the Petroleum Refining Industry (HJ2045-2014). The second draft of the

74 Emission Standard of Pollutants for Onshore Oil and Gas Exploitation and Production, which was

75 circulated for comments in 2018, represents a further attempt to enhance oil and gas industry 76 regulations. ${ }^{21}$

\section{$78 \quad$ FPW toxicity}

80 Survival, growth, and reproduction of aquatic animals Depending on FPW chemistry, raw

81 FPW samples have been shown to cause acute mortality (defined as a concentration that is lethal

82 to $50 \%$ of a population, or LC50) at FPW concentrations of $0.6 \%-3.9 \%$ (zebrafish embryos), ${ }^{24}$

83 and $11.6 \%$ (juvenile rainbow trout) ${ }^{22}$ in vertebrate aquatic species, and approximately $4 \%$ in

84 invertebrate aquatic species (Lumbriculus variegatus). ${ }^{25}$ Furthermore, exposure to FPW

85 concentrations as low as $0.04 \%$ has been shown to decrease the mobility and reproductive potential 
86 of aquatic species (e.g., Daphnia magna), ${ }^{26}$ and inhibit cardio-respiratory functioning and

87 swimming performance (e.g., in zebrafish). ${ }^{27}$

89 Functional and morphological impairment in exposed animals Embryonic exposure to a $2.5 \%$

90 FPW solution has been shown to have prolonged adverse effects on swimming performance in

91 juvenile zebrafish. ${ }^{27}$ Morphological studies in fish have also determined that FPW solutions as low

92 as $2.5 \%$ may induce certain developmental deformities such as pericardial edema, yolk-sac edema,

93 and tail/spine curvatures (in larval zebrafish), ${ }^{28}$ with gill abnormalities occurring in juvenile and

94 adult rainbow trout exposed to $2.5 \%-3 \%$ solutions of FPW. ${ }^{29,30}$

95

96 Endocrine disruptive property Human cell line reporter gene assays on HF chemicals,

97 contaminated surface and groundwater associated with HF activity, as well as real FPW samples

98 have demonstrated significant agonist and/or antagonist activities that disrupt the normal

99 functioning of estrogen, androgen, progesterone, glucocorticoid and thyroid hormone receptors. ${ }^{31-}$

$100{ }^{34}$ Impaired expressions of genes related to endocrine disruption (e.g., vtg, ar, era, erb, cyp19a,

101 etc.) have been reported in zebrafish and rainbow trout exposed to real FPW samples. ${ }^{34-37}$ Prenatal

102 exposure to an HF chemical mixture with endocrine-disrupting activity resulted in a variety of

103 reproductive and developmental health outcomes in male and female mice, including increased

104 weights of the body, heart, testes and thymus, decreased sperm counts and folliculogenesis,

105 suppressed sex steroid and pituitary hormones, and altered development of the uterine, ovary and

106 mammary glands. ${ }^{38-40}$

107 
108 FPW impact on microbial communities Findings show that aquatic microorganisms 109 Rheinheimera and Brevundimonas may be quickly enriched during FPW exposure, ${ }^{41,42}$ while the 110 abundance of Flavobacterium is significantly reduced. ${ }^{41}$ 


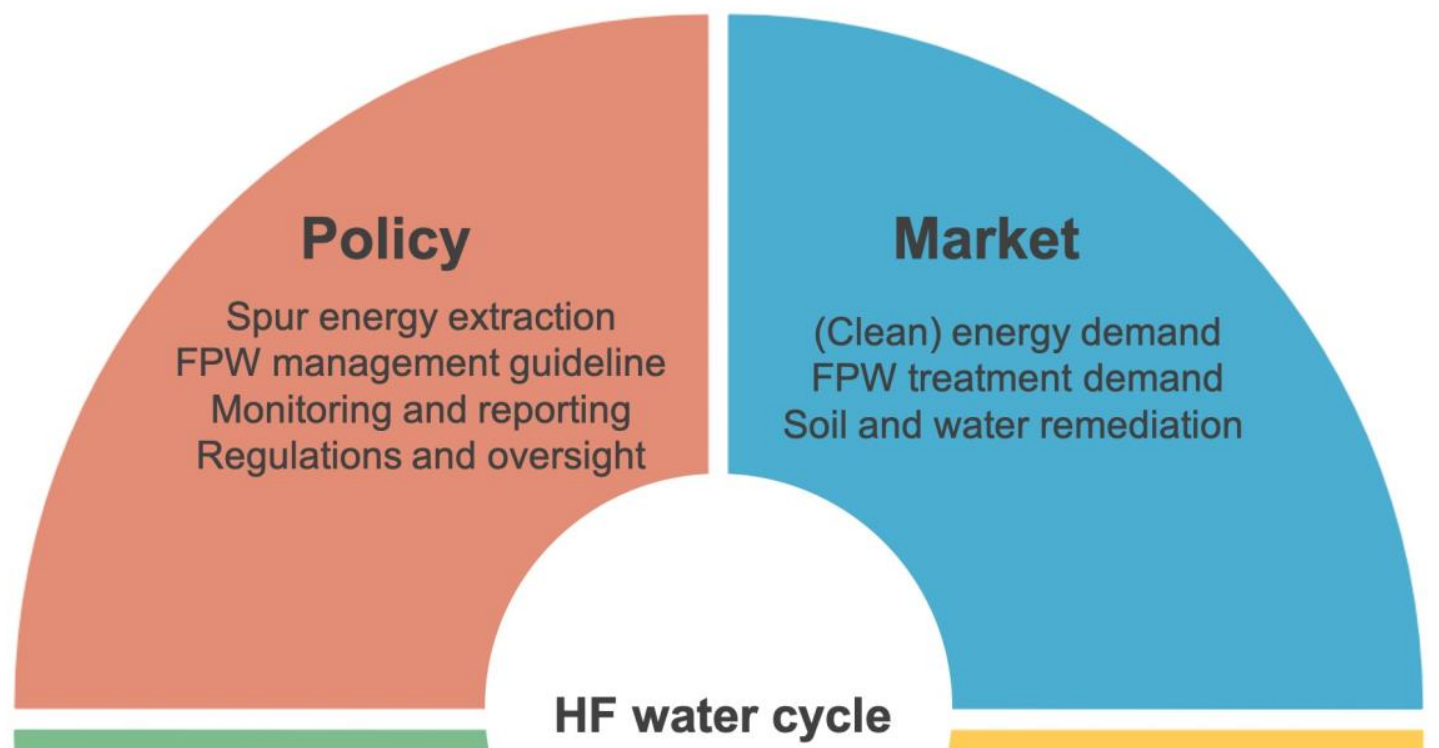

112
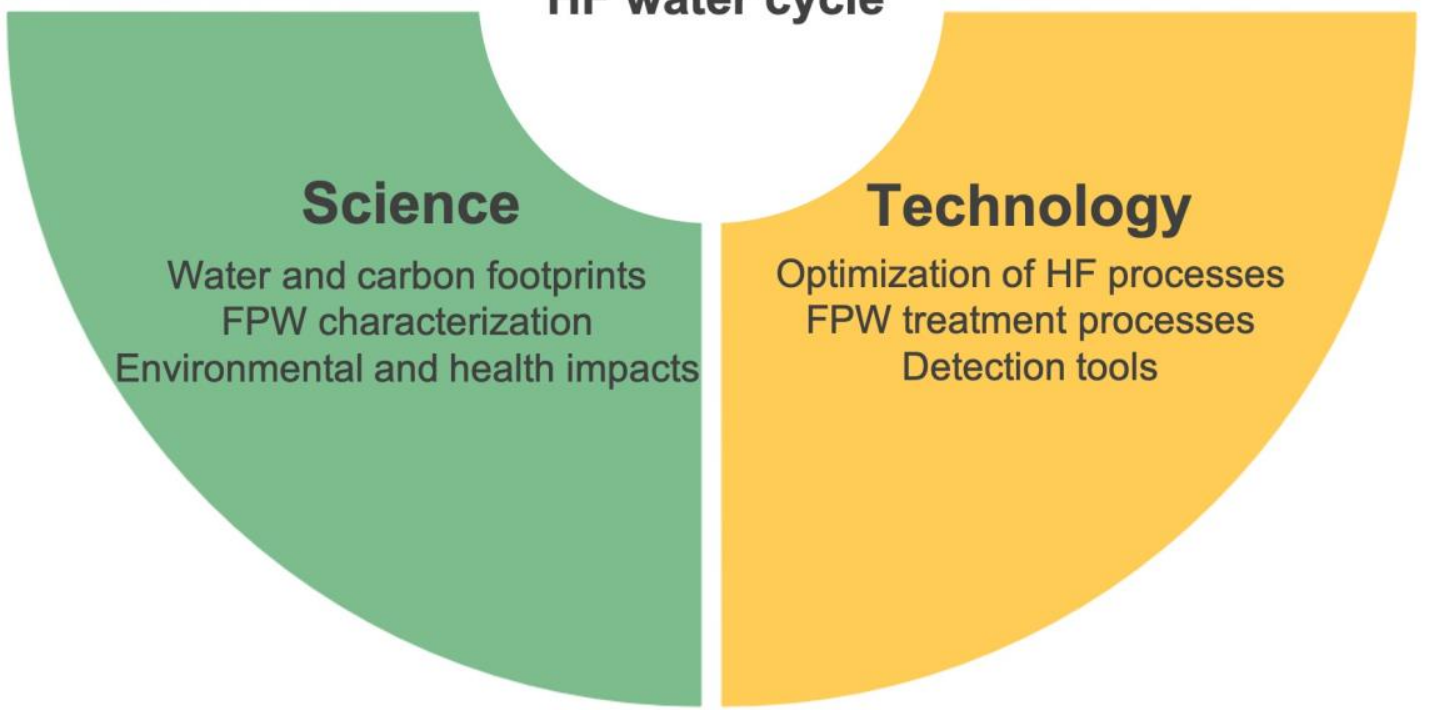

113 Figure S1. Illustration of how the HF water cycle interlinks with science, technology, policy, and

114 the market.

115 

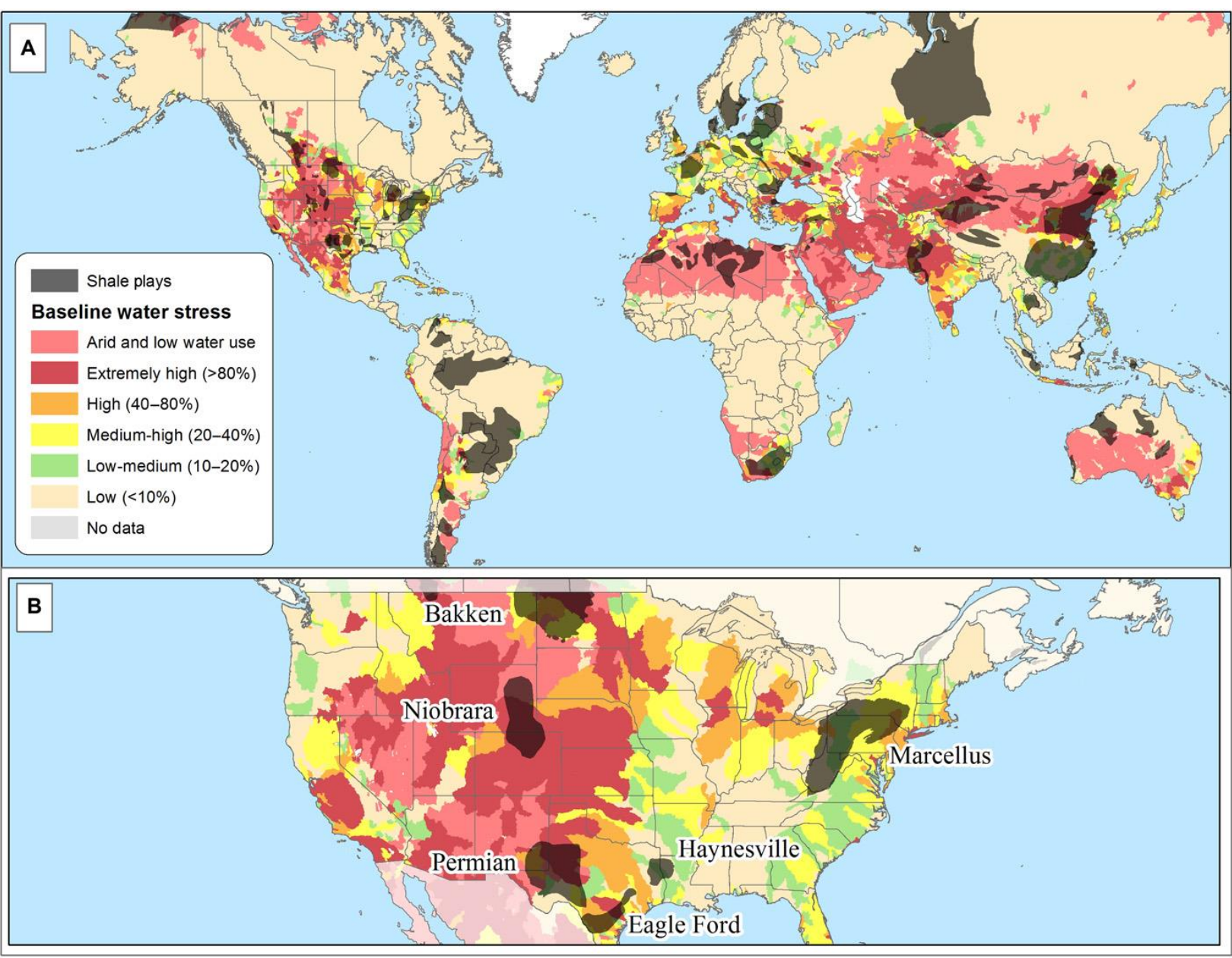

117 Figure S2. Global distribution of shale plays and water stress. Reprinted with permission from

118 Kondash et al. (2018). ${ }^{43}$ Copyright 2018 AAAS Science Advances. 


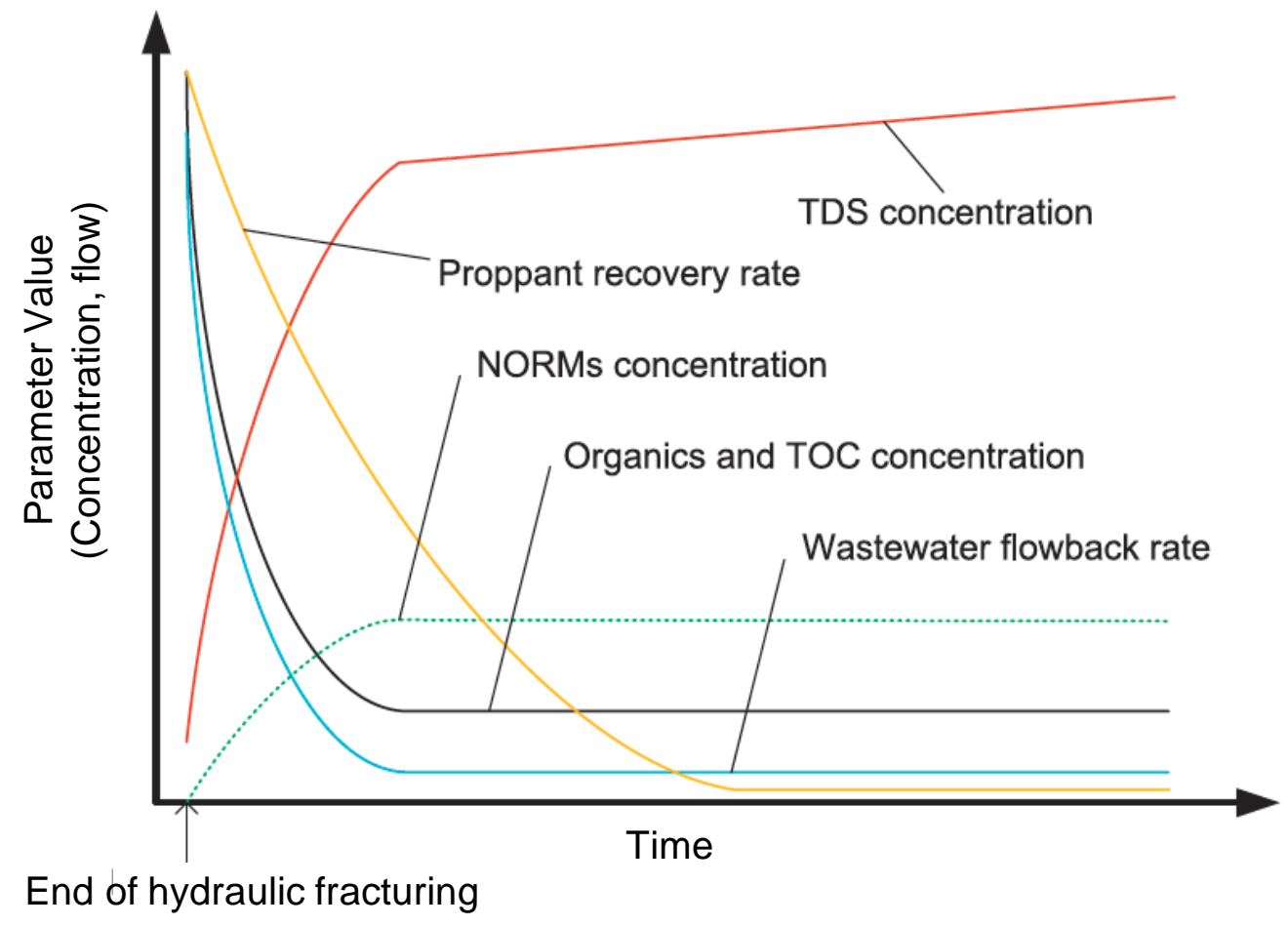

121 Figure S3. Conceptual diagram of parameters associated with FPW production after HF operations.

122 Reprinted with permission from Estrada and Bhamidimarri (2016). ${ }^{43}$ Copyright 2016 Elsevier 123 


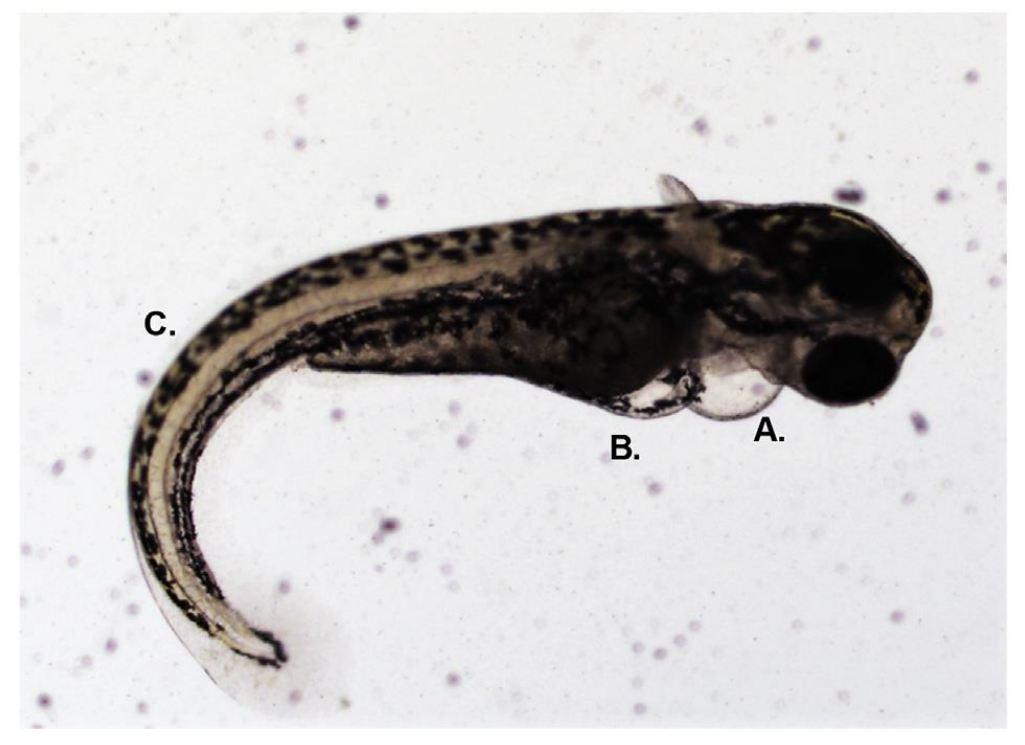

125 Figure S4. Photograph of measured developmental deformities in a larval zebrafish. Pericardial 126 edema (A), yolk-sac edema (B), and tail/spine curvatures (C) were measured in zebrafish $120 \mathrm{~h}$ 127 post-fertilization after acute embryonic (24 h post-fertilization) FPW exposure. The representative 128 organism shown in the image above was exposed $24 \mathrm{~h}$ post fertilization to $2.5 \%$ FPW-S for $48 \mathrm{~h}$. 129 Image was taken at $20 \times$ magnification. Reprinted with permission from Folkerts et al. (2016). ${ }^{43}$ 130 Copyright 2017 Elsevier 
133 Table S1. Raw data for Figure 3

\begin{tabular}{|c|c|c|c|}
\hline Natural gas type & Region & Year & Gas production (billion $\mathrm{m}^{3}$ ) \\
\hline \multirow[t]{37}{*}{ Total natural gas } & China $^{44}$ & 2007 & 69.24 \\
\hline & & 2008 & 80.299 \\
\hline & & 2009 & 85.269 \\
\hline & & 2010 & 95.791 \\
\hline & & 2011 & 105.337 \\
\hline & & 2012 & 110.608 \\
\hline & & 2013 & 120.858 \\
\hline & & 2014 & 130.157 \\
\hline & & 2015 & 134.61 \\
\hline & & 2016 & 136.865 \\
\hline & & 2017 & 148.035 \\
\hline & & 2018 & 160.265 \\
\hline & & 2019 & 176.17 \\
\hline & U.S. ${ }^{45}$ & 2007 & 545.552205 \\
\hline & & 2008 & 570.8271011 \\
\hline & & 2009 & 584.0015489 \\
\hline & & 2010 & 603.5869486 \\
\hline & & 2011 & 648.5079273 \\
\hline & & 2012 & 680.5451867 \\
\hline & & 2013 & 685.4229537 \\
\hline & & 2014 & 733.1107669 \\
\hline & & 2015 & 766.4072177 \\
\hline & & 2016 & 753.003602 \\
\hline & & 2017 & 773.2272624 \\
\hline & & 2018 & 866.1741568 \\
\hline & Canada $^{46}$ & 2007 & 173.5795447 \\
\hline & & 2008 & 166.6939945 \\
\hline & & 2009 & 155.7260257 \\
\hline & & 2010 & 150.7851871 \\
\hline & & 2011 & 150.9785261 \\
\hline & & 2012 & 143.1927616 \\
\hline & & 2013 & 145.4658744 \\
\hline & & 2014 & 152.9939715 \\
\hline & & 2015 & 156.5355532 \\
\hline & & 2016 & 157.7653556 \\
\hline & & 2017 & 160.3232687 \\
\hline & & 2018 & 166.6922031 \\
\hline
\end{tabular}




\begin{tabular}{|c|c|c|c|}
\hline Shale gas & & 2019 & 162.1772776 \\
\hline \multirow[t]{33}{*}{ Shale gas } & \multirow[t]{7}{*}{ China $^{47}$} & 2013 & 0.2 \\
\hline & & 2014 & 1.3 \\
\hline & & 2015 & 4.471 \\
\hline & & 2016 & 7.882 \\
\hline & & 2017 & 8.995 \\
\hline & & 2018 & 10.881 \\
\hline & & 2019 & 15.4 \\
\hline & \multirow[t]{13}{*}{ U.S. $^{45}$} & 2007 & 36.6136224 \\
\hline & & 2008 & 59.9183488 \\
\hline & & 2009 & 88.065248 \\
\hline & & 2010 & 151.0984448 \\
\hline & & 2011 & 226.3644992 \\
\hline & & 2012 & 293.6735328 \\
\hline & & 2013 & 323.236272 \\
\hline & & 2014 & 380.7760096 \\
\hline & & 2015 & 430.7834784 \\
\hline & & 2016 & 482.2917376 \\
\hline & & 2017 & 526.3809952 \\
\hline & & 2018 & 624.4987072 \\
\hline & & 2019 & 723.7459 \\
\hline & \multirow[t]{13}{*}{ Canada $^{46}$} & 2007 & 0 \\
\hline & & 2008 & 0.206736 \\
\hline & & 2009 & 0.723576 \\
\hline & & 2010 & 1.860624 \\
\hline & & 2011 & 4.341456 \\
\hline & & 2012 & 4.961664 \\
\hline & & 2013 & 6.408816 \\
\hline & & 2014 & 6.098712 \\
\hline & & 2015 & 5.271768 \\
\hline & & 2016 & 5.478504 \\
\hline & & 2017 & 5.375136 \\
\hline & & 2018 & 5.1684 \\
\hline & & 2019 & 4.961664 \\
\hline
\end{tabular}

134 
Table S2. Values for Figure 4 A-E

\begin{tabular}{|c|c|c|c|c|c|c|c|}
\hline Region & Basin & Location & $\begin{array}{c}\text { Water use per } \\
\text { well }\left(\mathrm{m}^{3} / \text { well, }\right. \\
2016)^{4,16,43,48-50}\end{array}$ & $\begin{array}{l}\text { Water use per } \\
\text { well per lateral } \\
\text { length }\left(\mathrm{m}^{3} / \mathrm{m} \text {, }\right. \\
2016)^{16,43,48-50}\end{array}$ & $\begin{array}{c}\text { FPW volume } \\
\left(\mathrm{m}^{3} / \text { well }\right)^{16,43,48-51}\end{array}$ & $\begin{array}{c}\text { No. of } \\
\text { wells } 16,43,48,49\end{array}$ & $\begin{array}{l}\text { Proved gas } \\
\text { reserves }^{7,52}\end{array}$ \\
\hline \multirow{2}{*}{ China } & & Changning & 45000 & 27.7 & 15000 & 148 & 136 \\
\hline & & Weiyuan & 34000 & 22.6 & 19800 & 97 & 27 \\
\hline \multirow[t]{4}{*}{ U.S. } & Appalachian & Marcellus & 27950 & 16.7 & 5200 & 8307 & 3826 \\
\hline & Permian Basin & Permian Basin & 42560 & 29.27 & 74471 & 33000 & 1322 \\
\hline & Western Gulf & Eagle Ford & 23457 & 12.76 & 6940 & 9411 & 872 \\
\hline & TX-LA Salt & Haynesville & 58777 & 32.75 & 17510 & 3172 & 1266 \\
\hline \multirow[t]{2}{*}{ Canada } & WCSB & Duvernay & $10000-5000$ & & 50000 & 1923 & \\
\hline & & Montney & $10000-5000$ & & 17500 & 1009 & \\
\hline
\end{tabular}

137 Note that data for Permian Basin and Eagle Ford pertain to shale gas. For unconventional oil wells in Eagle Ford in 2016, water use per

138 well was $33,492 \mathrm{~m}^{3} /$ well, water use per lateral length was $17.58 \mathrm{~m}^{3} / \mathrm{m}$, and FPW volumes were $15,119 \mathrm{~m}^{3} /$ per well. ${ }^{43}$ For unconventional

139 oil wells in the Permian Basin in 2016, water use per well was 42,560 $\mathrm{m}^{3} /$ well, water use per lateral length was $21.14 \mathrm{~m}^{3} / \mathrm{m}$, and FPW

140 volumes were $36,217 \mathrm{~m}^{3} /$ well. $^{43}$ 
141 Table S3. Values for Figure 4F

\begin{tabular}{|c|c|c|c|}
\hline Region & Basin & Location & Maximum TDS \\
\hline \multirow[t]{3}{*}{ China } & Sichuan Basin & Fuling ${ }^{53}$ & 65300 \\
\hline & & Changning ${ }^{48}$ & 38774 \\
\hline & & Weiyuan $^{54}$ & 53533 \\
\hline \multirow[t]{7}{*}{ U.S. } & Appalachian & Marcellus $^{55}$ & 476500 \\
\hline & Fort Worth & Barnett $^{55}$ & 300155 \\
\hline & Permian Basin & Permian Basin ${ }^{56}$ & 212984 \\
\hline & Western Gulf & Eagle Ford ${ }^{56}$ & 398024 \\
\hline & Appalachian & Utica $^{57}$ & 283000 \\
\hline & Williston Basin & Bakken $^{55}$ & 632689 \\
\hline & Denver Basin & Niobrara ${ }^{56}$ & 30600 \\
\hline \multirow[t]{2}{*}{ Canada } & WCSB & Duvernay $^{58}$ & 219243 \\
\hline & & Montney ${ }^{58}$ & 104000 \\
\hline
\end{tabular}

142

143 
144 Table S4. FPW treatment options reported by water source regions. The table has been modified

145 from Sun et al. (2019)..$^{59}$

\begin{tabular}{llll}
\hline \multicolumn{1}{c}{ Treatment } & \multicolumn{1}{c}{ Water source } & \multicolumn{1}{c}{ Method } & Cost \\
\hline MS & Fuling, China & Coagulation-UF & \\
& Marcellus, U.S. & MF & \\
& Barnett, U.S. & RO-UF & $\$ 18.4 / \mathrm{m}^{3}$ \\
\hline FO & Marcellus, U.S. & Ceramic MF & \\
& Haynesville, U.S. & EC-FO & \\
\hline MVC & Fayetteville, U.S. & EC-FO & $\$ 200 / \mathrm{m}^{3}$ \\
\hline EC & Utica, U.S. & MVC-FO & \\
\hline AOP & Denver-Julesburg, U.S. & EC-biochar & \\
\hline Adsorption & Southeastern Ordos Basin, China & nZVI-H ${ }_{2}$ & \\
& Changning, China & Activated zeolite & \\
\hline Biological & Denver-Julesburg, U.S. & Biochar & \\
& Marcellus, U.S. & Aerobic sludge & Aerobic activated sludge \\
& Shengli Oilfield, China & MFCs, MCDCs & \\
\hline
\end{tabular}

146 MS: membrane separation, FO: forward osmosis, MVC: mechanical vapor compression, EC:

147 electrocoagulation, AOP: advanced oxidization process, UF: ultrafiltration, MF: microfiltration,

148 RO: reverse osmosis, MFCs: microbial fuel cells, MCDCs: capacitive deionization cells 
149 Table S5. Guidelines/practices regarding concentrations of substances permitted for HF water treatment and reuse (units of mg/L with

150 exception of $\mathrm{pH}$ and total bacteria). These guidelines are implemented by local jurisdictions.

\begin{tabular}{|c|c|c|c|c|c|c|c|}
\hline $\begin{array}{l}\text { Parameter } \\
(\mathrm{mg} / \mathrm{L})\end{array}$ & $\begin{array}{c}\text { Reuse for } \\
\mathrm{HF}^{59}\end{array}$ & $\begin{array}{l}\text { Irrigation } \\
(\text { China })^{56}\end{array}$ & $\begin{array}{l}\text { Irrigation } \\
(\text { U.S. })^{56}\end{array}$ & $\begin{array}{l}\text { Irrigation } \\
{\text { (Canada })^{60}}^{\text {Can }}\end{array}$ & $\begin{array}{c}\text { Livestock } \\
\text { water (U.S.) }\end{array}$ & $\begin{array}{c}\text { Surface water } \\
\text { discharge } \\
(\text { China })^{56}\end{array}$ & $\begin{array}{c}\text { Surface water } \\
\text { discharge } \\
\text { (U.S.) }{ }^{56}\end{array}$ \\
\hline TDS & $\begin{array}{c}<50,000- \\
65,000\end{array}$ & 1,000 & 2,000 & $500-3,500$ & & & 500 \\
\hline TSS & $<50$ & 80 & 100 & & & 70 & \\
\hline Oil and grease & & 5 & 35 & & & 5 & 0.01 \\
\hline $\mathrm{pH}$ & $6-8$ & $5.5-8.5$ & $6.5-8.4$ & & & & \\
\hline $\begin{array}{l}\text { Total } \\
\text { hardness }\end{array}$ & 2,500 & & & & & & 1,000 \\
\hline COD & & 150 & & & & & \\
\hline $\mathrm{Cl}$ & $\begin{array}{c}<20,000- \\
30,000\end{array}$ & 350 & 350 & $100-700$ & & & 250,230 \\
\hline $\mathrm{SO}_{4}^{2-}$ & $<44.27$ & & 960 & & 1000 & & 250 \\
\hline $\mathrm{NH}_{4}-\mathrm{N}$ & & & 5 & & & 15 & 11.63 \\
\hline $\mathrm{Ca}$ & & & 400 & & & & \\
\hline $\mathrm{Mg}$ & & & 60 & & $250-500$ & & \\
\hline $\mathrm{Fe}$ & $<20$ & & 5 & 5 & & & 1 \\
\hline $\mathrm{Al}$ & & & 5 & 5 & 5 & & 0.087 \\
\hline $\mathrm{Pb}$ & & 0.2 & 5 & 0.2 & 0.1 & 1 & 0.2 \\
\hline $\mathrm{Cd}$ & & 0.01 & 0.01 & 0.01 & 0.05 & 0.002 & 0.1 \\
\hline $\mathrm{Hg}$ & & 0.001 & & & 0.01 & 0.05 & 0.001 \\
\hline $\mathrm{V}$ & & & 0.1 & 0.1 & 0.1 & & \\
\hline $\mathrm{Zn}$ & & 2 & 2 & $1-5$ & 24 & 2 & 0.12 \\
\hline $\mathrm{Se}$ & & 0.02 & 0.02 & 0.02 & 0.05 & & 0.035 \\
\hline $\mathrm{Ba}$ & & & & & & & 10 \\
\hline
\end{tabular}




\section{References}

154 (1) U.S. Environmental Protection Agency. The Hydraulic Fracturing Water Cycle; 2016.

155 (2) Rivard, C.; Lavoie, D.; Lefebvre, R.; Séjourné, S.; Lamontagne, C.; Duchesne, M. An Overview of Canadian Shale Gas Production and Environmental Concerns. Int. J. Coal Geol. 2014, 126, 64-76.

(3) Gregory, K. B.; Vidic, R. D.; Dzombak, D. A. Water Management Challenges Associated with the Production of Shale Gas by Hydraulic Fracturing. Elements 2011, 7 (3), 181-186.

(4) Alessi, D. S.; Zolfaghari, A.; Kletke, S.; Gehman, J.; Allen, D. M.; Goss, G. G. Comparative Analysis of Hydraulic Fracturing Wastewater Practices in Unconventional Shale Development: Water Sourcing, Treatment and Disposal Practices. Can. Water Resour. J. 2017, 42 (2), 105-121.

164 (5) Sun, C.; Zhang, Y.; Alessi, D. S.; Martin, J. W. Nontarget Profiling of Organic Compounds in A Temporal Series of Hydraulic Fracturing Flowback and Produced Waters. Environ. Int. 2019, $131,104944$.

(6) China National Energy Administration. National Energy Administration Circular on The Issuance of Shale Gas Development Plan (2016-2020) (In Chinese); 2016.

169 (7) Dong, D.; Zou, C.; Dai, J.; Huang, S.; Zheng, J.; Gong, J.; Wang, Y.; Li, X.; Guan, Q.; 170

(8) Zhu, R.; Zou, C.; Mao, Z.; Yang, H.; Hui, X.; Wu, S.; Cui, J.; Su, L.; Li, S.; Yang, Z. 178, 37-51. 
175 (9) Yang, Z.; Zou, C.; Wu, S.; Lin, S.; Pan, S.; Niu, X.; Men, G.; Tang, Z.; Li, G.; Zhao, J.; Jia, X. Formation, Distribution and Resource Potential of The "Sweet Areas (Sections)" of Continental Shale Oil in China. Mar. Pet. Geol. 2019, 102, 48-60.

(10) U.S. Energy Information Administration. Technically Recoverable Shale Oil and Shale Gas Resources: China; 2015.

180

(11) Nicot, J. P.; Scanlon, B. R. Water Use for Shale-Gas Production in Texas, U.S. Environ. Sci. Technol. 2012, 46 (6), 3580-3586.

182

(12) Entrekin, S.; Trainor, A.; Saiers, J.; Patterson, L.; Maloney, K.; Fargione, J.; Kiesecker, J.; Baruch-Mordo, S.; Konschnik, K.; Wiseman, H.; Nicot, J. P.; Ryan, J. N. Water Stress from High-Volume Hydraulic Fracturing Potentially Threatens Aquatic Biodiversity and Ecosystem Services in Arkansas, United States. Environ. Sci. Technol. 2018, 52 (4), 23492358.

(13) Ni, Y.; Zou, C.; Cui, H.; Li, J.; Lauer, N. E.; Harkness, J. S.; Kondash, A. J.; Coyte, R. M.; Dwyer, G. S.; Liu, D.; Dong, D.; Liao, F.; Vengosh, A. Origin of Flowback and Produced Waters from Sichuan Basin, China. Environ. Sci. Technol. 2018, 52 (24), 14519-14527.

(14) Safari, S.; Lottermoser, B. G.; Alessi, D. S. Metal Oxide Sorbents for The Sustainable Recovery of Lithium from Unconventional Resources. Appl. Mater. Today 2020, 19, 100638.

(15) Daly, R. A.; Borton, M. A.; Wilkins, M. J.; Hoyt, D. W.; Kountz, D. J.; Wolfe, R. A.; Welch, S. A.; Marcus, D. N.; Trexler, R. V; MacRae, J. D.; Krzycki, J. A.; Cole, D. R.; Mouser, P. J.; Wrighton, K. C. Microbial Metabolisms in A 2.5-km-Deep Ecosystem Created by Hydraulic Fracturing in Shales. Nat. Microbiol. 2016, 1, 16146.

(16) Shi, W.; Wang, X.; Guo, M.; Shi, Y.; Feng, A.; Liang, R.; Raza, A. Water Use for Shale 
Gas Development in China's Fuling Shale Gas Field. J. Cleaner. Prod. 2020, 120680.

(17) Lan, D.; Chen, M.; Liu, Y.; Liang, Q.; Tu, W.; Chen, Y.; Liang, J. Development of Shale Gas in China and Treatment Options for Wastewater Produced from the Exploitation: Sustainability Lessons from the United States. J. Environ. Eng. 2020, 146 (9).

(18) Wang, D.; Sun, Y.; Tsang, D. C. W.; Hou, D.; Khan, E.; Alessi, D. S.; Zhao, Y.; Gong, J.; Wang, L. The Roles of Suspended Solids in Persulfate/Fe2+ Treatment of Hydraulic Fracturing Wastewater: Synergistic Interplay of Inherent Wastewater Components. Chem. Eng. J. 2020, 388, 124243.

(19) Wang, D.; Sun, Y.; Tsang, D. C. W.; Khan, E.; Cho, D. W.; Zhou, Y.; Qi, F.; Gong, J.; Wang, L. Synergistic Utilization of Inherent Halides and Alcohols in Hydraulic Fracturing Wastewater for Radical-Based Treatment: A Case Study of Di-(2-Ethylhexyl) Phthalate Removal. J. Hazard. Mater. 2020, 384, 121321.

(20) Ministry of Ecology and Environment of the People's Republic of China. Integrated Wastewater Discharge Standard (GB 8978-1996) (In Chinese); 1996.

(21) Ministry of Ecology and Environment of the People's Republic of China. Emission Standard of Pollutants for Onshore Oil and Gas Exploitation and Production Industry: Second Draft for Comments (In Chinese); 2018.

(22) Guo, M.; Lu, X.; Nielsen, C. P.; McElroy, M. B.; Shi, W.; Chen, Y.; Xu, Y. Prospects for Shale Gas Production in China: Implications for Water Demand. Renewable Sustainable Energy Rev. 2016, 66, 742-750.

(23) Ministry of Ecology and Environment of the People's Republic of China. Environmental Quality Standards for Surface Water (In Chinese); 2002.

(24) He, Y.; Flynn, S. L.; Folkerts, E. J.; Zhang, Y.; Ruan, D.; Alessi, D. S.; Martin, J. W.; Goss, 
G. G. Chemical and Toxicological Characterizations of Hydraulic Fracturing Flowback and Produced Water. Water Res. 2017, 114, 78-87.

223

224

225

226

227

228

229

230

231

232

233

234

235

236

237

238

239

240

241

242

243

(25)

Mehler, W. T.; Nagel, A.; Flynn, S.; Zhang, Y.; Sun, C.; Martin, J.; Alessi, D.; Goss, G. G. Understanding the Effects of Hydraulic Fracturing Flowback and Produced Water (FPW) to The Aquatic Invertebrate, Lumbriculus Variegatus Under various Exposure Regimes. Environ. Pollut. 2020, 259, 113889.

(26) Blewett, T. A.; Delompré, P. L. M.; He, Y.; Folkerts, E. J.; Flynn, S. L.; Alessi, D. S.; Goss, G. G. Sublethal and Reproductive Effects of Acute and Chronic Exposure to Flowback and Produced Water from Hydraulic Fracturing on the Water Flea Daphnia magna. Environ. Sci. Technol. 2017, 51 (5), 3032-3039.

(27) Folkerts, E. J.; Blewett, T. A.; He, Y.; Goss, G. G. Alterations to Juvenile Zebrafish (Danio rerio) Swim Performance after Acute Embryonic Exposure to Sub-lethal Exposures of Hydraulic Fracturing Flowback and Produced Water. Aquat. Toxicol. 2017, 193, 50-59.

(28) Folkerts, E. J.; Blewett, T. A.; He, Y.; Goss, G. G. Cardio-Respirometry Disruption in Zebrafish (Danio rerio) Embryos Exposed to Hydraulic Fracturing Flowback and Produced Water. Environ. Pollut. 2017, 231 (Pt 2), 1477-1487.

(29) Delompré, P. L. M.; Blewett, T. A.; Snihur, K. N.; Flynn, S. L.; Alessi, D. S.; Glover, C. N.; Goss, G. G. The Osmotic Effect of Hyper-Saline Hydraulic Fracturing Fluid on Rainbow Trout, Oncorhynchus Mykiss. Aquat. Toxicol. 2019, 211, 1-10.

(30) Blewett, T. A.; Weinrauch, A. M.; Delompré, P. L. M.; Goss, G. G. The Effect of Hydraulic Flowback and Produced Water on Gill Morphology, Oxidative Stress and Antioxidant Response in Rainbow Trout (Oncorhynchus Mykiss). Sci. Rep. 2017, 7, 46582.

(31) Kassotis, C. D.; Tillitt, D. E.; Davis, J. W.; Hormann, A. M.; Nagel, S. C. Estrogen and 
Androgen Receptor Activities of Hydraulic Fracturing Chemicals and Surface and Ground Water in A Drilling-Dense Region. Endocrinology 2014, 155 (3), 897-907.

(32) Kassotis, C. D.; Iwanowicz, L. R.; Akob, D. M.; Cozzarelli, I. M.; Mumford, A. C.; Orem,

W. H.; Nagel, S. C. Endocrine Disrupting Activities of Surface Water Associated with A

West Virginia Oil and Gas Industry Wastewater Disposal Site. Sci. Total Environ. 2016, 557-558, 901-910.

(33) Kassotis, C. D.; Vu, D. C.; Vo, P. H.; Lin, C. H.; Cornelius-Green, J. N.; Patton, S.; Nagel,

S. C. Endocrine-Disrupting Activities and Organic Contaminants Associated with Oil and Gas Operations in Wyoming Groundwater. Arch. Environ. Contam. Toxicol. 2018, 75 (2), $247-258$.

He, Y.; Zhang, Y.; Martin, J. W.; Alessi, D. S.; Giesy, J. P.; Goss, G. G. In Vitro Assessment of Endocrine Disrupting Potential of Organic Fractions Extracted from Hydraulic Fracturing Flowback and Produced Water (HF-FPW). Environ. Int. 2018, 121, 824-831.

(35)

He, Y.; Sun, C.; Zhang, Y.; Folkerts, E. J.; Martin, J. W.; Goss, G. G. Developmental Toxicity of the Organic Fraction from Hydraulic Fracturing Flowback and Produced Waters to Early Life Stages of Zebrafish (Danio Rerio). Environ. Sci. Technol. 2018, 52 (6), 38203830.

(36) He, Y.; Folkerts, E. J.; Zhang, Y.; Martin, J. W.; Alessi, D. S.; Goss, G. G. Effects on Biotransformation, Oxidative Stress, and Endocrine Disruption in Rainbow Trout (Oncorhynchus Mykiss) Exposed to Hydraulic Fracturing Flowback and Produced Water. Environ. Sci. Technol. 2017, 51 (2), 940-947.

Blewett, T. A.; Delompré, P. L. M.; He, Y.; Folkerts, E. J.; Flynn, S. L.; Alessi, D. S.; Goss, G. G. Sublethal and Reproductive Effects of Acute and Chronic Exposure to Flowback and 
Produced Water from Hydraulic Fracturing on the Water Flea Daphnia magna. Environ. Sci.

Technol. 2017, 51 (5), 3032-3039.

(38) Kassotis, C. D.; Klemp, K. C.; Vu, D. C.; Lin, C. H.; Meng, C. X.; Besch-Williford, C. L.;

Pinatti, L.; Zoeller, R. T.; Drobnis, E. Z.; Balise, V. D.; Isiguzo, C. J.; Williams, M. A.;

Tillitt, D. E.; Nagel, S. C. Endocrine-Disrupting Activity of Hydraulic Fracturing Chemicals and Adverse Health Outcomes After Prenatal Exposure in Male Mice. Endocrinology 2015, 156 (12), 4458-4473.

Developmental Health Outcomes Following Prenatal Exposure to A Hydraulic Fracturing Chemical Mixture in Female C57B1/6 Mice. Endocrinology 2016, 157 (9), 3469-3481.

Sapouckey, S. A.; Kassotis, C. D.; Nagel, S. C.; Vandenberg, L. N. Prenatal Exposure to Unconventional Oil and Gas Operation Chemical Mixtures Altered Mammary Gland Development in Adult Female Mice. Endocrinology 2018, 159 (3), 1277-1289.

(41) Zhong, C.; Nesbø, C. L.; Goss, G. G.; Lanoil, B. D.; Alessi, D. S. Response of Aquatic Microbial Communities and Bioindicator Modelling of Hydraulic Fracturing Flowback and Produced Water. FEMS Microbiol. Ecol. 2020, 96, fiaa068.

(42) Kekacs, D.; Drollette, B. D.; Brooker, M.; Plata, D. L.; Mouser, P. J. Aerobic Biodegradation of Organic Compounds in Hydraulic Fracturing Fluids. Biodegradation 2015, 26 (4), 271-287.

(43) Kondash, A. J.; Lauer, N. E.; Vengosh, A. The Intensification of The Water Footprint of Hydraulic Fracturing. Sci. Adv. 2018, 4 (8), eaar5982.

Chinese National Bureau of Statistics. The Natural Gas Production by Years (In Chinese); 
2019.

(45) U.S. Energy Information Administration. How much shale gas is produced in the United States?; 2021.

(46) Government of Canada. Exploration and Production of Shale and Tight Resources; 2020.

Official Website of PetroChina Company Limited. Shale Gas Production in China (Chinese).

(48) Liu, D.; Li, J.; Zou, C.; Cui, H.; Ni, Y.; Liu, J.; Wu, W.; Zhang, L.; Coyte, R.; Kondash, A.;

Vengosh, A. Recycling Flowback Water for Hydraulic Fracturing in Sichuan Basin, China: Implications for Gas Production, Water Footprint, and Water Quality of Regenerated Flowback Water. Fuel 2020, 272, 117621.

(49) Kondash, A.; Vengosh, A. Water Footprint of Hydraulic Fracturing. Environ. Sci. Technol. Lett. 2015, 2 (10), 276-280.

(50) Zou, C.; Ni, Y.; Li, J.; Kondash, A.; Coyte, R.; Lauer, N.; Cui, H.; Liao, F.; Vengosh, A. The Water Footprint of Hydraulic Fracturing in Sichuan Basin, China. Sci. Total Environ. 2018, 630, 349-356.

(51) Goss, G.; Alessi, D.; Allen, D.; Gehman, J.; Brisbois, J.; Kletke, S.; Sharak, A. Z.; Notte, C.; Yvette, D.; Hong, K.; Renan, V.; Junes, C.; Barroso, W. Unconventional Wastewater Management: A Comparative Review and Analysis of Hydraulic Fracturing Wastewater Management Practices across Four North American Basins; 2015.

(52) U.S. Energy Information Administration. U.S. Crude Oil and Natural Gas Proved Reserves, Year-End 2018; 2019.

(53) Huang, T.; Pang, Z.; Li, Z.; Li, Y.; Hao, Y. A Framework to Determine Sensitive Inorganic Monitoring Indicators for Tracing Groundwater Contamination by Produced Formation 
Water from Shale Gas Development in the Fuling Gasfield, SW China. J. Hydrol. 2020, 581, 124403.

(54) Gao, J.; Zou, C.; Li, W.; Ni, Y.; Liao, F.; Yao, L.; Sui, J.; Vengosh, A. Hydrochemistry of Flowback Water from Changning Shale Gas Field and Associated Shallow Groundwater in Southern Sichuan Basin, China: Implications for the Possible Impact of Shale Gas

(55) Torres, L.; Yadav, O. P.; Khan, E. A Review on Risk Assessment Techniques for Hydraulic

Fracturing Water and Produced Water Management Implemented in Onshore Unconventional Oil and Gas Production. Sci. Total Environ. 2016, 539, 478-493.

Chang, H.; Li, T.; Liu, B.; Vidic, R. D.; Elimelech, M.; Crittenden, J. C. Potential and Implemented Membrane-Based Technologies for The Treatment and Reuse of Flowback 57.

Blondes, M. S.; Shelton, J. L.; Engle, M. A.; Trembly, J. P.; Doolan, C. A.; Jubb, A. M.; Chenault, J. C.; Rowan, E. L.; Haefner, R. J.; Mailot, B. E. Utica Shale Play Oil and Gas Brines: Geochemistry and Factors Influencing Wastewater Management. Environ. Sci. Technol. 2020, 54 (21), 13917-13925.

(58) von Gunten, K.; Snihur, K. N.; McKay, R. T.; Serpe, M.; Kenney, J. P. L.; Alessi, D. S. Characterizing Returning Polymers in Hydraulic-Fracturing Flowback and Produced Water: Implications for Colloid Formation. SPE J. 2020, 26(2): 563-590.

Sun, Y.; Wang, D.; Tsang, D. C. W.; Wang, L.; Ok, Y. S.; Feng, Y. A Critical Review of Risks, Characteristics, and Treatment Strategies for Potentially Toxic Elements in Wastewater from Shale Gas Extraction. Environ. Int. 2019, 125, 452-469. 
336 (60) Council of Ministers of the Environment. Canadian Water Quality Guidelines; 2008. 